\title{
Making Nutrition Services Work for Socially Excluded Groups: Lessons from the Integrated Nutrition and Health Project
}

\author{
Mukesh Kumar, Dora Warren, Sridhar Srikantiah, Sampurna \\ Singh, George Kurian, Mercy Manoranjini, Reetu Sharma and \\ Arundhuti Roy Choudhury
}

\begin{abstract}
A$ relatively large proportion of India's underweight children belong to groups facing multiple disadvantages. Addressing child malnutrition among these communities is critical if India is to eliminate undernutrition and achieve the MDG goals. This article draws evidence from the Integrated Nutrition and Health Project II (INHP-II), a USAID funded project, implemented by CARE in India, to show how, by ensuring universal service coverage, a programme can enhance equity and inclusion. INHP-approaches such as: Nutrition and health days (NHD); prioritising home contacts; system strengthening; community participation; tracking left-out children; enhancing convergence and coverage of nutritional and health services, all help to improve nutritional outcomes among all sections of society, particularly socially excluded groups.
\end{abstract}

\begin{abstract}
1 Social exclusion and undernutrition: the linkages

India is home to more than one-third of the world's undernourished children and the highest prevalence is among girls, Scheduled Castes (SC) and Scheduled Tribes (ST). ${ }^{1}$ The National Family Health Survey-3 (NFHS-3 2005-06)² (IIPS 2007) reveals that the percentages of underweight children under-five are higher among SC (47.9 per cent) and ST (54.5 per cent) than the non-scheduled groups (42.5 per cent). A comparison of nutritional status across various social groups between NFHS-2 (1998-9) (IIPS 2000) and NFHS-3 (2005-06) (IIPS 2007) indicates that stunting and wasting in children has worsened among SC, ST and Other Backward Caste (OBC) communities (NFHS $2 / 3)$. The rate of reduction in undernutrition has been relatively slower for girls than for boys. The Sachar committee report (CARE India 2006) highlighted disparities in nutritional status based on religion. Muslim children showed the highest rates of stunting. Child undernutrition
\end{abstract}

also has a strong correlation with the mother's education. The percentage of children that are severely underweight is five times higher among those whose mothers had no education than those where mothers had more than 12 years of education (NFHS-3; IIPS 2007). Undernutrition is more common in children of mothers with a low body mass index (below 18.5) showing a correlation between maternal and child nutritional status. On the wealth index, children from households with the lowest standard of living were twice as likely to be undernourished as those from higher wealth quintiles (NFHS-3; IIPS 2007).

Social identity based on gender, caste, religion, education, region and economic status, are critical determinants of children's nutritional status and of their access to nutrition services. Caste is often a barrier to service access. Studies of the Integrated Child Development Scheme (ICDS), ${ }^{3}$ a Government of India (GoI), Ministry of Women and Child Development (MWCD) 
programme, show that many SC and ST communities are unable to access the programme services, because a large proportion of Anganwadi Centers (AWC) are located in the areas of dominant castes or religious groups. In India, the SC tend to live in geographically separate habitations outside or on the edge of larger settlements and are not able to access services and resources, such as water, in higher caste areas. Expanding the reach of services goes hand in hand with quality. There are instances where socially excluded groups choose to self exclude from accessing programme services, as they perceive providers to be less responsive to their needs (Mander 2006). In addition, socially marginalised groups such as ST and SC tend to live in areas/hamlets which are remote and under-served, leading to spatial inequality.

Drawing on the evidence from the Integrated Nutrition and Health Project II (INHP-II) designed on the platform of ICDS, operationalised by CARE and funded by United States Agency International Development (USAID), this article shows how INHP enabled the nutrition services to expand their reach to all sections of the population, particularly the socially excluded, using a set of strategies that: tracked left-out groups, improved health and nutrition service coverage and changed feeding practices.

\section{Project background}

The goal of the INHP-II (2001-06) was to achieve sustainable improvement in the nutrition and health status of women and children in nine states of India. ${ }^{5}$ It was implemented in 747 ICDS blocks in 78 districts across nine states. The project worked with pregnant and lactating women and children under two years of age. It adopted a twin-track approach, i.e. strengthening existing Government of India ICDS and Reproductive and Child Health (RCH) programmes and engaging communities. These approaches were tailored to local situations, thus making it possible for the project to reach socially excluded groups (CARE India 2008a).

\section{Framework for analysis}

The evidence for this article is mainly drawn from the project baseline - 2001 and endline 2006 and different rounds of rapid annual assessments (RAP I-2003, RAP II-2004, RAP III-
2005) conducted during the project (CARE India 2008b). Unfortunately, it was not possible to collect data on control groups, to construct a before and after, with and without design. Hence, the results in this article are suggestive, and far from conclusive. For the purpose of comparison, social and economic disaggregated data from INHP-II endline and NFHS-3 is used for some key nutrition related indicators.

Our analysis is restricted to three determinants of exclusion, namely caste, educational status and socioeconomic status (SES). The socioeconomic score is a composite index calculated in each INHP survey taking into account variables like caste, maternal education, maternal employment, type of housing, access to toilet facilities, electricity and potable water. The article analyses project outreach to different groups not only on the basis of this composite indicator of SES but also separately on the basis of caste and education. On the basis of caste as one of the determinants, two relative groups SC/ST and 'other castes' are compared. On the education level, a group of mothers with less than five years of education is compared with those with more than six years education. On SES, those with low SES are compared with others from a higher SES group. For a detailed profile of respondents, refer to Table 1.

Those with low SES, SC/ST and with education less than five years are referred as 'socially excluded', whereas those with high SES, other caste groups and those with more than six years education are 'the comparison group' in this article.

To understand improvements in health and nutrition service coverage, data on indicators, such as: (1) Women receiving 90+ iron folic acid (IFA) during pregnancy; (2) percentage of children (12-23 months) completely immunised; and (3) percentage of children (12-23 months) that received Vitamin A (1st dose), are analysed. To understand the changes in feeding practices, indicators such as: (1) Early initiation of breastfeeding; and (2) timely initiation of complementary feeding have been analysed. Data on important INHP processes, such as identifying the left-out groups, prioritised home contacts and nutrition and health day (NHD) are also analysed to show how the INHP interventions reached all sections of the 
Table 1 Incidence of nutrition behaviours at IHNP baseline (BL) and endline (EL)

\begin{tabular}{|c|c|c|c|c|c|c|c|c|c|c|c|c|}
\hline & \multicolumn{4}{|c|}{ Mother's education } & \multicolumn{4}{|c|}{ Caste } & \multicolumn{4}{|c|}{ Socioeconomic status } \\
\hline & \multicolumn{2}{|c|}{$0-5$ years } & \multicolumn{2}{|c|}{$>5$ years } & \multicolumn{2}{|c|}{$\mathrm{SC} / \mathrm{ST}$} & \multicolumn{2}{|c|}{ Others } & \multicolumn{2}{|l|}{ Low } & \multicolumn{2}{|l|}{ High } \\
\hline & $\mathrm{BL}$ & $\mathrm{EL}$ & $\mathrm{BL}$ & $\mathrm{EL}$ & $\mathrm{BL}$ & EL & $\mathrm{BL}$ & $\mathrm{EL}$ & $\mathrm{BL}$ & $\mathrm{EL}$ & $\mathrm{BL}$ & EL \\
\hline $\begin{array}{l}\text { Women reporting initiation of } \\
\text { breast-feeding within } 2 \text { hours of } \\
\text { birth (\%) }\end{array}$ & 26.0 & 39.0 & 20.1 & 37.4 & 29.9 & 40.9 & 18.7 & 39.4 & 29.1 & 41.5 & 20.0 & 36.5 \\
\hline $\begin{array}{l}\text { Children initiated semi solids } \\
\text { between } 6-8 \text { months of age (\%) }\end{array}$ & 55.9 & 61.2 & 59.0 & 64.5 & 56.6 & 63.7 & 60.8 & 56.4 & 56.4 & 64.0 & 57.0 & 60.2 \\
\hline $\begin{array}{l}\text { Women reported receiving } 90+ \\
\text { IFA tablets during pregnancy (\%) }\end{array}$ & 20 & 49 & 27 & 53 & 22 & 52 & 21 & 49 & 21 & 49 & 23 & 51 \\
\hline $\begin{array}{l}\text { Children } 12-23 \text { months receiving } \\
\text { complete immunisation (\%) }\end{array}$ & 37.0 & 54.6 & 63.6 & 74.5 & 36.2 & 58.8 & 45.0 & 59.8 & 34.1 & 55.7 & 47.0 & 63.2 \\
\hline $\begin{array}{l}\text { Children } 12-23 \text { months that } \\
\text { received vitamin A, 1st dose (\%) }\end{array}$ & 32.2 & 50.7 & 48.1 & 59.0 & 32.0 & 52.5 & 38.8 & 52.6 & 32.7 & 52.6 & 37.6 & 54.0 \\
\hline
\end{tabular}

IFA, iron folic acid.

population to contribute to equitable improvement in service coverage and feeding practices.

The following section discusses changes in health and nutritional services and feeding practices during the project period among different caste, SES and educational groups in order to understand the relative changes across categories of different determinants.

\section{Project results}

INHP promoted two key messages: (1) early and exclusive breast-feeding of children 0-6 months and (2) appropriate complementary feeding with emphasis on timely initiation, frequency, quantity and variety of food to be fed to the child.

\subsection{Early breast-feeding}

At the project baseline, the practice of early initiation of breast-feeding was 26 per cent (education less than five years, 29.9 per cent (SC/ST) and 29.1 per cent for lowest SES group). By the project endline, a 12.4 percentage point increase in the practice among those with low SES and an increase of 16.5 percentage points among those with higher SES is seen. Analysing the percentage change exclusively on the basis of caste, an 11 per cent increase in SC/ST and a 20.7 per cent increase in those from other castes is seen. On the education parameter, about 13 percentage points increase among both categories is seen. Thus, during the project period, there is an 11-13 per cent increase in those socially excluded and an increase of 13-20.7 per cent in the comparison group. Nonetheless, by the end of the project, the difference in achievement on this indicator between the socially excluded and comparison group had reduced to 1.5 per cent.

\subsection{Time initiation of complementary feeding}

In comparison to the early initiation of breastfeeding, the practice of timely initiation of complementary feeding was found to be more than 55 per cent among all categories of caste, education and composite indicator of SES at the project baseline (Table 1). The practice was slightly better among those from the comparison group than those socially excluded at the baseline. By the project endline, the improvement among SC/ST and low SES surpassed other castes and the higher SES group. Among both the educational categories, the percentage improvement was almost the same (5.3 and 5.5 per cent).

Similar trends in improvement were observed in coverage and delivery of health and nutritional service across groups. 


\subsection{Receipt of 90+ IFA tablets}

The project baseline data show that the coverage of $90+$ IFA tablets to women during pregnancy was generally low with only 20 per cent of those who had less than five years of education receiving IFA tablets; 22.4 per cent (SC/ST) and 21.1 per cent (low SES). It was also low in the comparison group (Table 1). Poorer, less educated and SC women were less likely to be given IFA tablets according to the baseline. By the project endline, there was a sharp increase in service coverage by around 28-29 per cent among pregnant women from socially excluded groups across caste, education and SES. There was an equal increase (26-29 per cent) among women from the comparison groups. By the end of the project, the proportion of women receiving $90+$ IFA had doubled.

\subsection{Complete immunisation}

Immunisation coverage showed consistent improvement (Table 1). When the project started, the proportion of those in the lowest SES who were completely immunised was 13 per cent less than those in the higher SES group. It was 8.8 per cent less among those from SC/ST caste groups than others. The gap was greatest between different educational categories. Those with less than five years of education were 26.6 per cent less likely to be completely immunised than those with more than six years of education. By the project endline, these gaps were reduced to 7.7 per cent among SES groups, 1 per cent among caste groups and 19.9 per cent among educational categories.

\subsection{Receipt of vitamin $A$ (1st dose)}

Receipt of Vitamin A (1st dose) among children 12-23 months reveals a similar pattern (Table 1). At project baseline, the proportion of children with low SES was 5 per cent less covered than those with high SES. The proportion of children from SC/ST was 7 per cent less covered than those from other castes. This inequity was greatest between those with more and less than six years' education (16 per cent). By the project endline, this inequity is reduced to almost negligible levels across categories of caste and SES. The gap between the two educational categories had halved.

A comparative analysis of INHP II endline (2006) and NFHS-3 (2005-6), also confirms that service coverage and practices such as the initiation of breast-feeding was more equitable and nutritional outcomes were better in districts covered by INHP. What led to this more equitable reach and uptake of services? The following section discusses the strategies that brought about this change.

\section{What led to this change?}

INHP was built on the existing platform of the Government's ICDS and RCH programmes. The equitable reach of health and nutritional services seen above was not a result of stand alone INHP initiatives. It was the outcome of combined and coordinated work between INHP and government programmes. INHP identified the key limitations and challenges of ICDS and RCH programmes, and adopted corrective measures to improve the quality and service coverage particularly to socially excluded groups.

Based on the INHP-I experience, the project's second phase adopted 'innovate-demonstrateadvocate-replicate' approaches. Some of the key practices identified for demonstration and replication are:

- Tracking and inclusion of those who were left out or dropped out from the service net.

- Nutrition and health days ${ }^{6}$ (NHD) to promote the coordinated functioning of frontline workers from health and ICDS, increase predictability and improve the coverage of health and ICDS services delivered on the same day and same site.

- Change agents/community volunteers from each habitation identified, appointed and trained to ensure that all are reached with essential services and correct messages.

- Community based monitoring systems (CBMS) to promote the active participation of community as a stakeholder to ensure that noone is left out and services reach all.

- Block level resource mapping (BLRM) demonstrated for its potential with support from partner NGOs (CARE India 2008c).

Capacity building (classroom and ongoing) of programme functionaries including frontline workers on issues such as: identifying who is left out; the causes of malnutrition including gender and social inclusion; counselling and monitoring of proper care and feeding practices; tracking women and children in need during critical times, i.e. pregnancy, lactation, infancy and 
Table 2 Home contacts made by service providers ( $A W W$, ANM, LHV) during the last trimester of pregnancy and first year of child's life

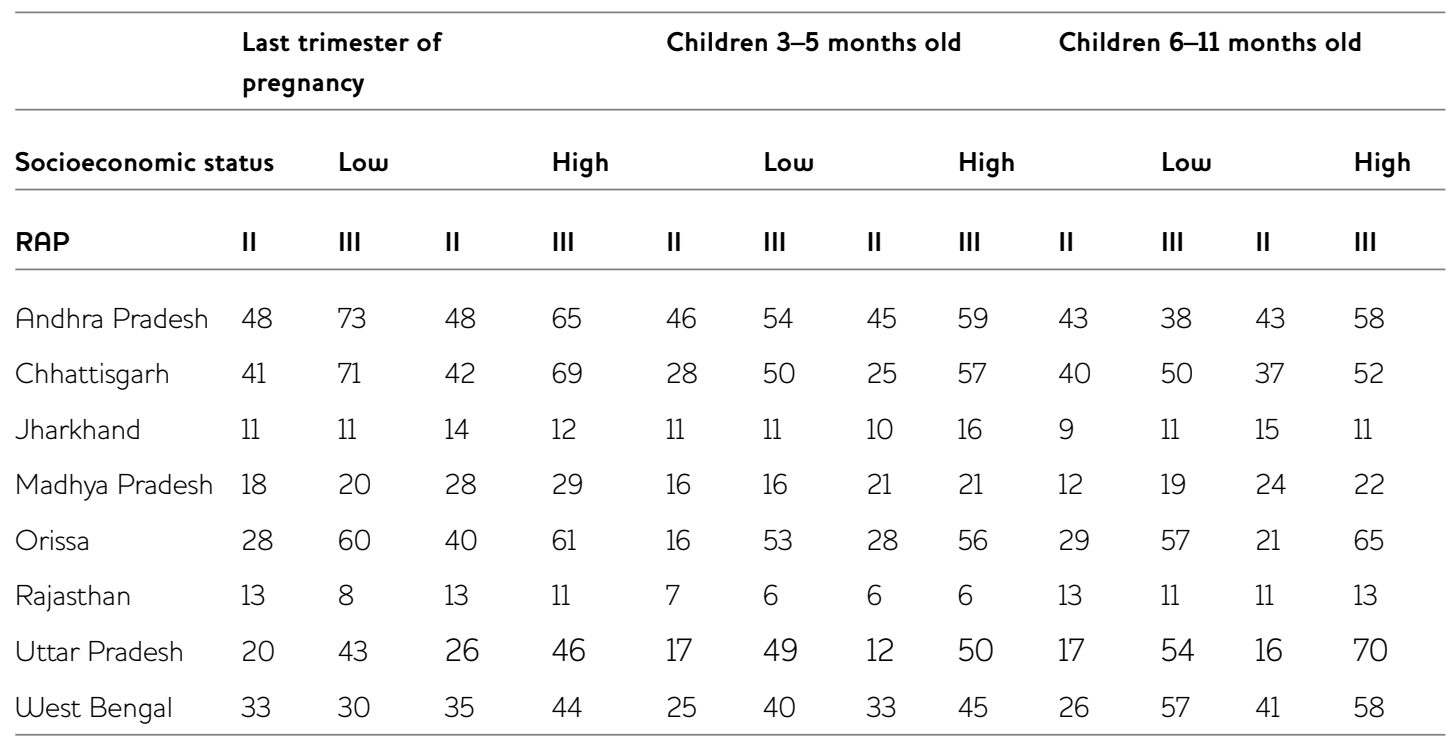

AWW, Anganwadi worker; ANM, auxiliary nurse midwifes; LHV, lady health visitor, a Supervisor appointed by the Health Department to supervise ANMs; RAP, rapid annual assessment

childhood, prioritising home contacts ${ }^{7}$ to those in need and delivering the right messages at the right time to the right person, the importance of convergence at all levels, the role of supportive supervision and community involvement were topics addressed by capacity building.

To implement these approaches, strategies included: (1) Development of tools such as 'Home Visit Planners ${ }^{\circ}$ registers with ready reckoners ${ }^{9}$ to prioritise home contacts and delivery of messages; (2) Due lists ${ }^{10}$ were designed to identify and track those due for services on an NHD; (3) Continuous ongoing training of community/hamlet volunteers to mobilise communities to demand services and build greater accountability of service providers was undertaken; (4) Mechanisms such as social mapping, participation of communitybased organisations (CBO) and Panchayati Raj Institutions ${ }^{11}$ (PRI) in NHDs and introducing health and nutrition agenda in Gram Sabha ${ }^{12}$ under CBMS were put in place.

In order to re-orient the programme focus on the ground, streamlining supervision at sector level was emphasised. Monthly sector meetings ${ }^{13}$ included time for ongoing capacity building of frontline workers on health, nutrition, gender and social inclusion issues apart from the routine administrative work. A supervisors' field visit tool $^{14}$ and a sector meeting checklist ${ }^{15}$ helped to make supervision more systematic. Participation of auxiliary nurse midwifes (ANMs) in the sector meetings was encouraged to establish convergence between ICDS and health programmes. ICDS and health officials at block and district levels were encouraged to conduct joint field visits and participate in monthly or quarterly reviews in consultation with other stakeholders, including local partner NGOs and PRIs. To institutionalise all these changes, there was further engagement with state and national level officials through programme reviews and advocacy (CARE India 2008c).

The project monitoring data reveals that the INHP process, similar to service delivery and feeding practices, reached both the socially excluded and the comparison groups. A comparison of home contacts data in different time periods across two SES categories show an increase from RAP II and RAP III. Four out of the eight INHP states show an increase from 23 to 32 per cent in home contacts made in the last trimester of pregnancy to those from low SES as compared with 17-27 percentage points increase 


\begin{tabular}{lll}
\hline & Baseline (\%) & Endline (\%) \\
\hline Andhra Pradesh & 54.9 & 66.0 \\
Chhattisgarh & 77.5 & 85.5 \\
Jharkhand & 33.7 & 75.4 \\
Madhya Pradesh & 77.7 & 76.6 \\
Orissa & 68.7 & 78.6 \\
Rajasthan & 10.3 & 53.8 \\
Uttar Pradesh & 15.4 & 57.7 \\
Uest Bengal & 4.8 & 13.4 \\
\hline
\end{tabular}

in those from high SES groups (Table 2). These home contacts were critical in terms of preparing mothers and families for birth, with messages on the 'five cleans' (clean place, clean surface, clean hands, clean cord and dressing, clean tie), safe delivery, thermal care, early and exclusive breast-feeding, no pre-lacteals and other practices associated with better pregnancy outcomes.

Similarly, home contacts made to mothers of children three to five months of age belonging to low SES show an increase of 8 to 37 per cent in five out of the eight INHP states compared to 14 to 38 percentage points increase among those with high SES (Table 2). These contacts are crucial to inform mothers about exclusive breastfeeding, timely initiation of complementary feeding, and the need for complete immunisation.

Another important period for home contact is when the child reaches $6-11$ months of age. Here again, four out of the eight INHP states show an increase of 10-37 percentage points among those with low SES and an increase of 15-54 percentage points among those with high SES (Table 2). The importance of home visits at this period of life is towards promoting appropriate complementary feeding, complete immunisation and Vitamin A supplementation.

The causal relationship between improved home contacts and service coverage and feeding behaviours is hard to prove, but an equitable increase in home contacts is associated with an equitable increase in service coverage and feeding behaviours.
INHP also promoted the NHD strategy. This involved the distribution of supplementary food (ICDS) and health services together on the same day at the AWC. In order to assess the reach and effectiveness of this process, data on the proportion of children completely immunised whose mothers received supplementary food (SF) during their pregnancy and lactation period vs. those who did not receive it, has been analysed (Table 3). Across INHP states, the proportion of children completely immunised was higher when mothers had received SF from an AWC during lactation than those who did not receive SF. The home contacts, regular tracking and registration by AWWs facilitated the receipt of SF on an NHD. With the emphasis on convergent functioning of both ANM and AWWs, there is a strong correlation between those contacted by an AWW for ICDS services and the home contacts made by the ANM (CARE India 2008d). Data shows that those who received SF on an NHD were more likely to access immunisation and other services and they were more likely to be poor. Supplementary food acted as an incentive for poorer women to access essential health and nutrition services for their children.

\section{Conclusion}

As noted earlier, the evaluation of IHNP is not a true impact study due to the absence of control groups. Nevertheless, because (a) the outcomes (coverage) are so tightly tied to the intervention (improved outreach) and (b) the increases in changed behaviours and utilisation are so large and unidirectional, the plausibility of impact is high. It would take major non-intervention events to increase outreach to this extent - these may have occurred, but they were not observed. 
Nevertheless, by ensuring universal coverage of services and by joining forces with multiple stakeholders, INHP was probably responsible for improving the reach of nutrition and health programmes to the whole community and especially those who are more likely to be excluded from such programmes.

One of the key lessons is that maximising programme outreach with the right messages at the right time to the right people improves coverage and social inclusion. Joining forces with multiple stakeholders INHP was able to improve nutrition and health programme reach among all categories but more particularly the socially excluded group who generally constituted a large proportion of the left-out groups. INHP innovations such as tracking those who were left out, prioritising home visits, more responsive service provision through NHD and the selection of volunteer change agents for each hamlet helped to improve inclusion. Inclusion of those who were previously left out, increased the overall coverage and improved the outcomes of the programme.

Equally important was community mobilisation and monitoring, partnership with local NGOs, community's participation in programme planning and review of performance by the system, supportive supervision and strengthened

\section{Notes}

* The views expressed in this article do not necessarily represent those of DFID.

1 Scheduled Castes (SC) and Scheduled Tribes (ST) are Indian population groupings that are explicitly recognised by the Constitution of India, previously called the 'depressed classes' by the British, and otherwise known as untouchables. SCs/STs together comprise over 24 per cent of India's population, with SC at over 16 per cent and ST at over 8 per cent as per the 2001 Census.

2 The National Family Health Survey (NFHS) is a large-scale, multi-round survey conducted in a representative sample of households throughout India. Three rounds of the survey have been conducted since the first survey in 1992-3. The survey provides state and national information for India on fertility, infant and child mortality, the practice of family planning, maternal and child health, reproductive health, nutrition, anaemia, convergent sector, block and district level review meetings.

INHP processes were applied at scale in different contexts, although tailored to meet local context and needs. Importantly, what worked for general context also befitted those socially excluded, thus resulting in better inclusion and reduced inequity in terms of access to quality services. In other words, processes and strategies used in INHP-II can be scaled up and integrated in the newly proposed universalisation of the ICDS programme working at scale.

The essence of convergent models promoted by INHP like NHD, sector, block and district level meetings to enhance service coverage and promote behavioural change are already in place under the National Rural Health Mission (NRHM). The provision of ASHA (Accredited Social Health Activist) in each village under NRHM is another opportunity. These, together with the convergent functioning of the three frontline workers - AWW, ANM and ASHA with adequate supportive supervision focused on inclusion tracking, prioritised home visits and more responsive service provision, would be able to better address exclusion. Now with the onset of ICDS reforms and NRHM in India, the convergent strategies and functioning can enhance service outreach, inclusion and nutrition outcomes.

utilisation and quality of health and family planning services. Each successive round of the NFHS has had two specific goals: (1) to provide essential data on health and family welfare needed by the Ministry of Health and Family Welfare and other agencies for policy and programme purposes, and (2) to provide information on important emerging health and family welfare issues.

3 ICDS, the national government programme started in 1975. It is operational in 4,200 ICDS projects (in India, a block is an administrative unit under district with a population of $100,000)$. At the village level, the project is implemented through Anganwadi workers (AWW) and Anganwadi helpers (AWH) in an Anganwadi Centre (AWC). There is a Lady Supervisor (LS) at the Sector level, a level between AWC and block that supervises the AWW functions. LS supervise 4-5 AWCs and AWWs. This number is now increased under the universalisation of ICDS. At the project or 
block level, the project functions are overseen by Child Development Project Officers (CDPO). The administrative functions related to the programme are seen by various officials at district, state and national levels. The principal participants of the scheme are: children below six years, expectant and nursing mothers and women in the age group of 15-45 years. The package of services include health check-ups; immunisation; referral services; provision of supplementary nutrition, growth monitoring and promotion; nutrition and health education to women; early childhood care and pre-school education.

4 Anganwadi Centre is an institution of ICDS at village level which is operated by Anganwadi workers (AWW) to provide all prescribed nutrition and health services of ICDS to the target groups.

5 The nine states where INHP II was implemented were: Andhra Pradesh (AP), Bihar (BH), Chhattisgarh (CG), Jharkhand (JH), Madhya Pradesh (MP), Orissa (OR), Rajasthan (RJ), Uttar Pradesh (UP) and West Bengal (WB). Since the programme was only implemented in Bihar in October 2004, the results shared are from only eight states.

6 By project definition, on NHD, both an AWW and an ANM must be present and both supplementary nutrition in the form of Take Home Rations (THR) and health services, namely antenatal services, immunisation and health and nutrition counselling, must be provided to the pregnant and lactating women and children.

7 Prioritised home contacts: the strategy emphasised ICDS workers to make home contacts to those most in need first. This identification of who to visit first was facilitated by the use of home visit planners and registers. The decision is made on the basis of criticality of the period in life cycle and criticality of current health and nutritional status of the woman or child at that point in time.

8 Home Visit Planner - Register, is a structured tool which is used by an AWW in order to plan her daily home visits. It minimises the bias of the AWW to visit any home based on her convenience, as the planner has different columns for different categories of women and children on the basis of their month of pregnancy or age of their child. According to the protocol, there are prescribed numbers of home visits that are to be made by an AWW in each of these categories, thus minimising the chance of exclusion.

9 Ready reckoner is a systematic behavioural change guide that is attached to the home visit planner. This guide has all the messages that the AWW must convey during her home visit to the pregnant or lactating mother of children 0-23 months of age towards better health and nutritional behaviours.

$10 \mathrm{~A}$ Due list is the final list made by the ANM and AWW after consulting their survey registers and lists. This final list includes the names of children due for immunisation with various vaccines. This tally mechanism thus helps in tracking the left-outs or drop-outs and promotes maximum inclusion.

11 Panchayat/Panchayati Raj Institution (PRI): The three-tier institution of local self-governance system in India consisting of the elected representative bodies at the district, block and village levels is called PRI.

12 Gram Sabha/Gramm sansad is the assembly of all voters in the jurisdiction of a village where villagers are given the opportunity to raise their concerns as part of the system of governance under PRIs. It is mandated to meet twice a year.

13 Sector meetings: monthly, one day ICDS meetings (in some states, the meeting is for two days), conducted at the sector where all the AWWs under the respective lady supervisor meet for a monthly review and update on the programme. Ongoing capacity building on health and nutrition issues is also part of the sector meetings in INHP states.

14 Supervisory field visit tool: the tool is designed to structure the monthly prescribed number of field visits made by ICDS lady supervisors. The tool covers all areas an LS is expected to observe, monitor and supervise and provides feedback to AWWs. The reported observations from the field visits using this tool facilitate the supportive supervision and feedback to the AWWs.

15 Sector meeting checklist: the list of agenda and issues necessary for discussion in every sector meeting. This listing facilitates time management of a one-day-long sector meeting by identifying all important issues, prioritising, allocating time for discussion and making a plan of action for the following month. 


\section{References}

CARE India (2008a) Paper 1: What RACHNA has Done So Far: Program Description, New Delhi, India, www.careindia.org (accessed 15 May 2009)

CARE India (2008b) Paper 2: Methods used for Assessment in the RACHNA Program, New Delhi, India, www.careindia.org

CARE India (2008c) Paper 10: Working with Existing Systems: Lessons from INHP, New Delhi, India, www.careindia.org

CARE India (2008d) Paper 6: Supplemental Feeding: Its Role in a Large Scale Maternal and Child Nutrition and Health Program, New Delhi, India, www.careindia.org
CARE India (2006) Sachar committee report. Status of Indian Muslims. Government of India, www.godgraces.org/files/

Muslim\%20Report.pdf (accessed 29 May 2009) International Institute for Population Sciences (IIPS) (2007) National Family Health Survey (NFHS-3) India 2005-06, Bombay: IIPS and ORC MACRO, Calverton, Maryland, USA

International Institute for Population Sciences (IIPS) (2000) National Family Health Survey (NFHS-2) India 1998-99, Bombay: IIPS and ORC MACRO, Galverton, Maryland, USA Mander, Harsh and Kumaran, M. (2006) 'Social Exclusion in ICDS: A Sociological Whodunit?', unpublished 